\section{Niveles de hemoglobina glicosilada y diferencia en el gasto en salud de pacientes diabéticos: un estudio econométrico}

RONY LENZ1,a, ALDO ZÁRATE², JORGE RODRÍGUEZ ${ }^{1, \mathrm{~b}}$, JORGE RAMÍREZ ${ }^{1}$

\section{Levels of glycosylated hemoglobin and the difference in the cost of health care for diabetic patients: an econometric study}

Background: Complications increase treatment costs of diabetes mellitus (DM). An adequate metabolic control of the disease could reduce these costs. Aim: To evaluate the costs of medical care for a cohort of patients with DM, according to their degree of metabolic compensation. Material and Methods: All diabetic patients attended in a regional hospital from 2005 to 2010 were analyzed. A correlational study between costs of individual healthcare and levels of glycosylated hemoglobin ( $\mathrm{HbA1c})$, was performed in a series of annual cross-sectional measurements. Results: The study comprised 1,644 diabetic patients. During the study period the average cost of healthcare per patient increased from $\$ 878,000$ to more than $\$ 1,000,000$ Chilean pesos (CLP) during the study period. The percentage of patients with $\mathrm{HbA}$ 1c levels below $7.0 \%$ varied between $43.0 \%$ and 54.9\%. Costs for patients with HbA1c levels between 7 and $8.9 \%$ were 1.3 to 1.5 times greater. For the group of patients with HbA1c levels between 9 and $10.9 \%$ the costs increased 1.4 to 1.6 times. For patients with HbAlc levels greater than $11.0 \%$, healthcare costs doubled. Conclusions: Healthcare expenditure varied according to metabolic control, which is consistent with international findings. This study was limited by its selected population, incomplete information on health expenditures, and the inclusion of only direct costs to the health system. If all patients would achieve metabolic compensation, the yearly savings would be CLP \$308,000,000 (or USD \$657,000).

(Rev Med Chile 2014; 142: 841-849)

Key words: Diabetes mellitus; Health care costs; Hemoglobin A, glycosylated.
${ }^{1}$ Escuela de Salud Pública. Universidad de Chile. Santiago de Chile.

${ }^{2}$ Hospital del Cobre Dr. Salvador Allende G. Calama, Chile.

aEconomista.

bestadístico.

Conflictos de intereses: Esta investigación fue financiada con un Grant Educacional del Laboratorio Sanofi-Aventis, quien no tuvo influencia en el diseño del estudio, en la recolección, análisis o interpretación de los datos; en la preparación, revisión o aprobación del manuscrito. El autor R. Lenz declaró recibir honorarios como Consultor del Laboratorio Sanofi-Aventis y del Instituto de Salud Pública y un subsidio a su institución por la International Society for Pharmacoeconomics and Outcome Research, Chilean Chapter; el autor A. Zárate declaró recibir honorarios como "Personal Assistant"; los autores J. Rodríguez y J. Ramírez recibieron honorarios de Lenz Consultores.

Recibido el 19 de abril de 2013 , aceptado el 30 de junio de 2014.

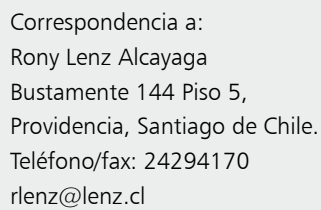

L a diabetes mellitus (DM) presenta retos extraordinarios para los sistemas de salud, no sólo por su alta prevalencia y la morbilidad que conlleva, sino por la complejidad y costos que implica su tratamiento.

Las proyecciones de prevalencia han ido en aumento desde la década pasada ${ }^{1}$. Se estima que cerca de $8,3 \%$ de la población mundial adulta presenta DM (unos 366 millones). Esto aumentará a 9,9\% (unos 552 millones) para el año $2030^{2}$. El año 2004 murieron 3,4 millones por la enfermedad. La Organización Mundial de la Salud (OMS) espera que estas cifras se dupliquen entre 2005 y 2030. Más de $80 \%$ de las muertes por DM ocurre en países de ingresos bajos y medios ${ }^{3}$.

En Chile, la prevalencia de la enfermedad es 9,4 por cien habitantes ${ }^{4}$, significativamente mayor que la pesquisada el año 2003 , de $6,3^{5}$. Se observa 
una cifra mayor en hombres y una marcada gradiente según el nivel educacional: personas con menos de 8 años de estudio más que triplican en prevalencia a personas con más de 12 años de educación. Existen 756.287 pacientes diabéticos en el seguro público (FONASA) y 65.368 en seguros privados (ISAPRE) ${ }^{6}$. Cerca de $70 \%$ de los primeros se encuentra bajo control en el sistema público de salud (SNSS), principalmente mujeres ${ }^{7}$. El total de diabéticos conocidos representaría casi dos tercios del total de diabéticos estimados para el país 4 .

La medición de hemoglobina glicosilada Alc (HbAlc) es el criterio estándar para la evaluación del control metabólico, que busca disminuir o retrasar la aparición de complicaciones micro y macro vasculares ${ }^{8,9}$. Se utilizan puntos de corte para considerar compensados a los pacientes. Chile utiliza $7 \%{ }^{10}$, el mismo de Estados Unidos de Norteamérica ${ }^{11}$. Otras recomendaciones exigen $6,5 \%{ }^{12}$. De los pacientes en control en los servicios públicos de salud en Chile, $38,6 \%$ presenta HbAlc consideradas adecuadas ${ }^{13}$, cifra similar a $34,3 \%$ detectado en encuestas poblacionales ${ }^{4}$. En promedio, el período en que los pacientes alcanzan compensación, desde el inicio de tratamiento, es 8 meses. La frecuencia de medición de $\mathrm{HbAlc}$ se encuentra bajo lo recomendado ${ }^{14}$.

Sostenidamente aumenta la prevalencia de DM, la complejidad del tratamiento y los costos del cuidado ${ }^{15-17}$. Estos costos varían entre países, debido a los métodos usados para estimarlos, a diferencias en práctica clínica, a distinto poder adquisitivo y al efecto de competencia con otras prioridades sanitarias ${ }^{18}$. Se suma el envejecimiento, individual y poblacional, que determina aumento en el gasto total en salud ${ }^{19-21}$. Un diabético cuesta hasta 2,5 veces el costo de un no diabético ${ }^{22,23}$, incluyendo pacientes hospitalizados ${ }^{24-26}$. Pacientes considerados prediabéticos, que progresan a DM tipo 2, aumentan sus costos de atención ${ }^{27}$, los que podrían ser efectivamente evitados ${ }^{28}$.

El número de complicaciones aumenta el costo de atención: la literatura anglosajona calcula costos directos según presencia de complicaciones ${ }^{29-31}$. Enfermos que presentan complicaciones micro y macro vasculares, o comorbilidad con hipertensión, pueden tener costos de atención 250\% más altos que pacientes diabéticos sin estas complicaciones $^{32-34}$. Lógicamente, factores que determinen un retraso en el diagnóstico o compensación, o que aumenten la necesidad de hospitalización, son elementos que impactan finalmente en la utilización de recursos del sistema de salud ${ }^{24}$. Los costos de pacientes diabéticos representan una alta proporción del gasto total de los sistemas de salud; algunas estimaciones los sitúan entre 7,0\% ${ }^{35}$ y $11,6 \%{ }^{36}$.

Intervenciones previas a la aparición de complicaciones en pacientes diabéticos (prevención secundaria efectiva) y un tratamiento adecuado (buen control metabólico), disminuyen la progresión de las complicaciones, la probabilidad de desarrollar enfermedades asociadas y la mortalidad $^{37-43}$, así como las atenciones médicas requeridas ${ }^{44,45}$. Se ha determinado la consecuente disminución de los costos directos, cuando estas intervenciones se dan en contexto de programas integrales de manejo de la enfermedad ("disease management") o de base comunitaria ${ }^{46-48}$. El retorno sobre la inversión (ROI) puede ser mayor a 4 veces $^{47}$.

Con información a nivel individual, este trabajo presenta una evaluación de gastos en atenciones sanitarias, asociados a presencia de DM según grado de compensación metabólica, en una cohorte cautiva de pacientes pertenecientes a una ISAPRE cerrada y atendidos en un servicio médico institucional.

\section{Pacientes y Métodos}

\section{Población bajo estudio}

La muestra correspondió al total de pacientes diabéticos controlados por el Hospital del Cobre (HDC) Dr. Salvador Allende G., en Calama, Chile, durante el período 2005 a 2010. Los criterios de inclusión fueron: 1) Existencia de diagnóstico clínico de DM, y 2) Disponibilidad de resultados de los exámenes de HbAlc al menos durante el último año.

\section{Control metabólico}

Según el promedio anual de HbAlc de cada año, se categorizó cada 2 puntos de incremento en HbAlc (por conveniencia y utilidad clínica), en: i) Controlado con HbAlc $<7,0 \%$; ii) No Controlado con $\mathrm{HbA1C}$ entre $7,0 \%$ y 8,9\%; iii) No controlado con HbA1C entre $9,0 \%$ y $10,9 \%$; y iv) No controlado con $\mathrm{HbA} 1 \mathrm{C}$ mayor o igual a $11,0 \%$.

\section{Gasto en salud}

El conjunto de prestaciones electivas y de 
urgencias, realizadas por el HDC a cada paciente durante el período, valorizadas en función del arancel fijado en el contrato de compra entre la ISAPRE y el HDC, correspondiente al año 2010, basado en codificación del Arancel de la Modalidad de Libre Elección de FONASA. A esto se sumó el gasto anual en farmacia, corregido por variación promedio del IPC anual. El gasto fue expresado en moneda nacional (\$) de junio de 2011.

Se precisa que el gasto presentado incluye procedimientos como diálisis, fotocoagulación y otros oftalmológicos terapéuticos. Se excluyen cirugías mayores debido a dificultad de acceso a información, manejada en registros históricos manuales. Tampoco se consideraron gastos en salud realizados fuera del HDC (gastos en Libre Elección o derivaciones institucionales a otro prestador). Este último tipo de gastos excluidos representan cerca de 10\% del total del gasto total del hospital, y corresponden a especialidades médico-quirúrgicas inexistentes o de baja resolutividad en el hospital (oncología, neurocirugía y traumatología).

\section{Diseño del estudio}

Serie de tiempo de mediciones transversales, que incluyó análisis correlacional de gastos en salud versus control metabólico, no contemplándose explicar la varianza del gasto. Para el análisis de regresión múltiple, primero se realizó análisis bivariado que permitiera ingresar en el modelo variables que fueran significativas. Se consideraron tres casos: 1) Modelo 1, dicotomizando la variable entre grupo controlado con $\mathrm{HbAlc}$
$<7 \%$ y pacientes no controlados con $\mathrm{HbAl}>$ $6,9 \%$; 2) Modelo 2, dicotomizando la variable entre el grupo controlado con $\mathrm{HbAlc}<7 \%$ y los pacientes no controlados con HbAlc $>8,9 \%$; y, 3) Modelo 3, dicotomizando la variable entre el grupo controlado con HbAlc $<7 \%$ y no controlado $\mathrm{HbA} 1 \mathrm{c}>10,9 \%$. Se consideró significativo un error máximo inferior al 0,05 (valor $\mathrm{p}<0,05$ ). $\mathrm{El}$ análisis de resultados se trabajó con paquetes estadísticos estándar.

\section{Recolección de la información}

Los datos fueron obtenidos desde bases de datos consolidadas a través del sistema informático del hospital. La atención cerrada se integró a la ficha clínica informatizada a partir de marzo de 2005.

\section{Resultados}

Se estudió una población de 1.644 diabéticos, que corresponden a $6,7 \%$ de la población adulta atendida en el HDC, con promedio de edad de 59,4 años (rango entre 19 y 94). El 53\% de los pacientes fueron hombres. El mayor porcentaje de pacientes diabéticos presentó $\mathrm{HbA1c}$ inferiores a 7,0\%. Este porcentaje ha ido en aumento desde 43,0\% (2005) a 54,9\% (2010). Los pacientes con HbAlc entre 7 y $8,9 \%$ se mantuvieron relativamente estables en alrededor de 32,0\%, excepto el año 2007 (42,4\%). Los segmentos con HbAlc entre 9 y 10,9\% y más de $11 \%$ han bajado sostenidamente en el período, desde $17,5 \%$ a $9,5 \%$ y desde $7,7 \%$ a $3,0 \%$, respec-

Tabla 1. Número de pacientes según tramos de resultados de HbA1c, Hospital del Cobre 2005-2010, en términos absolutos y porcentuales

\begin{tabular}{|c|c|c|c|c|c|c|}
\hline & 2005 & 2006 & 2007 & 2008 & 2009 & 2010 \\
\hline \multicolumn{7}{|l|}{ HbA1c } \\
\hline$<7,0 \%$ & 268 & 453 & 384 & 628 & 648 & 716 \\
\hline $7,0-8,9 \%$ & 198 & 266 & 419 & 345 & 420 & 426 \\
\hline $9,0-10,9 \%$ & 109 & 111 & 127 & 96 & 121 & 124 \\
\hline$>11,0 \%$ & 48 & 47 & 58 & 34 & 28 & 39 \\
\hline Total & 623 & 877 & 988 & 1.103 & 1.217 & 1.305 \\
\hline \multicolumn{7}{|l|}{ HbA1c } \\
\hline$<7,0 \%$ & $43,0 \%$ & $51,7 \%$ & $38,9 \%$ & $56,9 \%$ & $53,2 \%$ & $54,9 \%$ \\
\hline $7,0-8,9 \%$ & $31,8 \%$ & $30,3 \%$ & $42,4 \%$ & $31,3 \%$ & $34,5 \%$ & $32,6 \%$ \\
\hline $9,0-10,9 \%$ & $17,5 \%$ & $12,7 \%$ & $12,9 \%$ & $8,7 \%$ & $9,9 \%$ & $9,5 \%$ \\
\hline$>11,0 \%$ & $7,7 \%$ & $5,4 \%$ & $5,9 \%$ & $3,1 \%$ & $2,3 \%$ & $3,0 \%$ \\
\hline
\end{tabular}




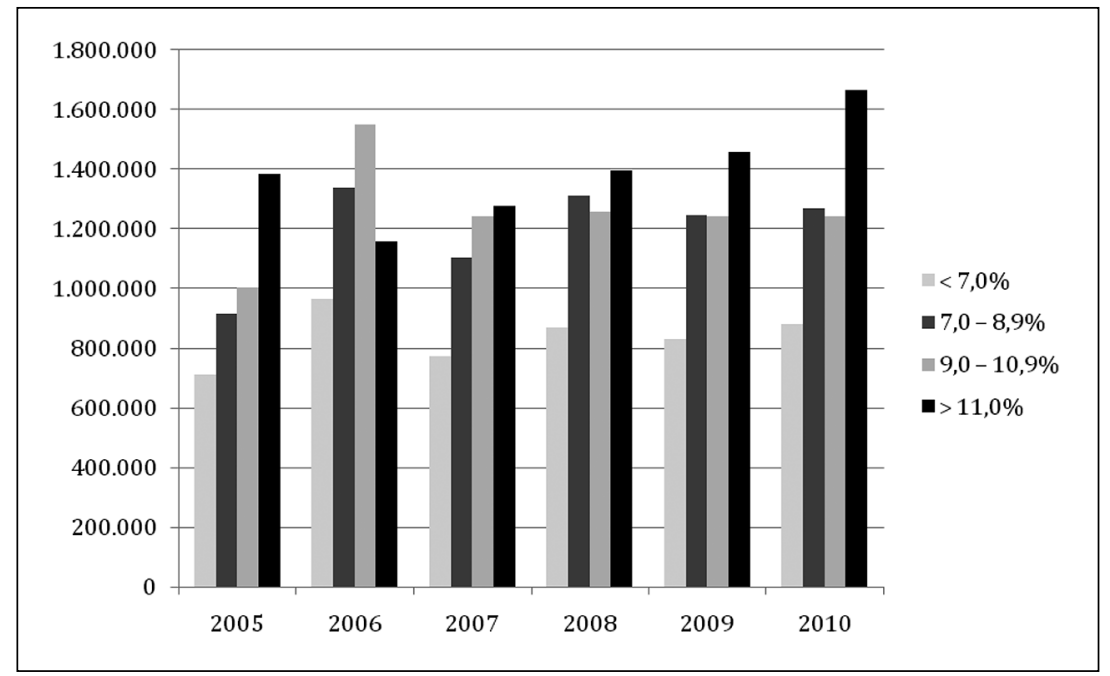

Figura 1. Gasto Medio en Salud por Pacientes según Tramos de Resultados de HbA1c, en términos absolutos, Hospital del Cobre 2005-2010, (Valores en \$ de junio 2011) tivamente (Tabla 1).

El gasto medio en salud por paciente se ha incrementado sostenidamente entre 2005 y 2010, desde $\$ 878.000$ a más de $\$ 1.000 .000$, esto es $21,3 \%$ acumulado (datos no mostrados). Este crecimiento ha sido similar en los diferentes tramos de pacientes agrupados por $\mathrm{HbAlc}$, excepto en el tramo de pacientes con HbAlc entre 7 y $8,9 \%$ cuyo gasto promedio se ha incrementado $38,7 \%$ (Figura 1).

Cuando se analiza cómo varía el gasto medio en salud de los diferentes tramos en relación al gasto asociado con los pacientes controlados (HbAlc $<7,0 \%$ ), se observa que el gasto del tramo con HbAlc entre 7 y $8,9 \%$ es entre 1,3 y 1,5 veces mayor, y algo similar sucede con el tramo con $\mathrm{HbAlc}$ entre 9 y 10,9\% ( 1,4 y 1,6 veces). En el tramo con HbAlc superiores a $11,0 \%$ el gasto puede llegar a duplicarse.

En los modelos de correlación (Tabla 2) se aprecia, a partir de valores del test $t$ de Student, que en todos los modelos y en todos los años la variable asociada al control metabólico es estadísticamente significativa (valor $\mathrm{p}<0,05$ ), exceptuando el coeficiente del modelo 3 en el año 2006 (valor $\mathrm{p}=0,21$ ). La variable edad no resultó significativa en análisis bivariado (se excluyó del análisis de regresión). La variable sexo resultó significativa en la mayoría de los análisis bivariados, aunque no en todos los años.

En las estimaciones de gastos de pacientes compensados y no compensados, de acuerdo a mode- los de regresión obtenidos (Tabla 3), se observa que existe mayor gasto asociado a pacientes no controlados en relación con aquellos controlados. En el modelo 1 (pacientes controlados vs todos los pacientes no controlados) el gasto en salud promedio de pacientes no controlados es $54 \%$ mayor en el caso de hombres y $43 \%$ en mujeres. En el caso del modelo 2 (pacientes controlados vs aquellos con $\mathrm{HbAlc}>8,9 \%$ ) el mayor gasto promedio es de $60 \%$ en hombres y $50 \%$ en mujeres. En el modelo 3 (pacientes controlados con no contralados vs pacientes con HbAlc > 10,9\%) el incremento promedio en el gasto es $76 \%$ en el caso de hombres y $63 \%$ para mujeres.

\section{Discusión}

Los resultados obtenidos en pacientes diabéticos que se atienden en el HDC (período 2005-2010), muestran que el gasto en salud ha aumentado en los últimos años, lo que se condice con la literatura, avalando costos crecientes de esta patología, en contexto con el envejecimiento poblacional $^{15-21}$.

Existe diferencia estadísticamente significativa en este gasto según grado de control metabólico. Esto es consistente con estudios que encuentran diferencias de costos basados en presencia de complicaciones propias de $\mathrm{DM}^{32-34}$. Al comparar un grupo sin complicaciones con un grupo con 
Costos en salud en pacientes diabéticos con mal control - R. Lenz et al

Tabla 2. Resultados modelos de correlación entre gasto en salud y control de pacientes diabéticos del Hospital del Cobre (valores de Beta y error estándar, en pesos chilenos a junio de 2011, para cada modelo y año)

Modelo con Variable Dicotómica: Grupo controlado (HbA1c < 7\%) y no controlado (HbA1 > 6,9\%)

\begin{tabular}{|c|c|c|c|c|c|c|c|c|c|}
\hline \multirow[t]{2}{*}{ Año } & \multicolumn{3}{|c|}{ Constante } & \multicolumn{3}{|c|}{ HbA1c } & \multicolumn{3}{|c|}{ Sexo } \\
\hline & B & Std. Error & $\mathbf{t}$ & B & Std. Error & $\mathbf{t}$ & B & Std. Error & $\mathbf{T}$ \\
\hline 2005 & 927.854 & 103.000 & 9,008 & 311.195 & 112.067 & 2,777 & -410.875 & 111.451 & $-3,687$ \\
\hline 2006 & 1.115 .891 & 81.552 & 13,683 & 417.174 & 92.144 & 4,527 & -281.509 & 92.626 & $-3,039$ \\
\hline 2007 & 854.361 & 68.195 & 12,528 & 379.979 & 72.123 & 5,268 & -147.001 & 70.597 & $-2,082$ \\
\hline 2008 & 953.687 & 66.913 & 14,253 & 450.678 & 79.303 & 5,683 & -173.133 & 78.985 & $-2,192$ \\
\hline 2009 & 877.337 & 64.291 & 13,646 & 431.991 & 74.922 & 5.766 & -93.931 & 75.104 & $-1,251$ \\
\hline 2010 & 876.554 & 64.535 & 13,583 & 414.496 & 95.358 & 4,347 & & & \\
\hline
\end{tabular}

Modelo con Variable Dicotómica: Grupo controlado (HbA1c < 7\%) y no controlado (HbA1 > 8,9\%)

\begin{tabular}{|c|c|c|c|c|c|c|c|c|c|}
\hline \multirow[t]{2}{*}{ Año } & \multicolumn{3}{|c|}{ Constante } & \multicolumn{3}{|c|}{ HbA1c } & \multicolumn{3}{|c|}{ Sexo } \\
\hline & B & Std. Error & $\mathbf{t}$ & B & Std. Error & $\mathbf{t}$ & B & Std. Error & $\mathbf{T}$ \\
\hline 2005 & 928.420 & 127.721 & 7,269 & 433.681 & 160.789 & 2,697 & -411.949 & 156.094 & $-2,639$ \\
\hline 2006 & 1.126 .687 & 82.060 & 13,73 & 485.289 & 117.247 & 4,139 & -301.413 & 103.566 & $-2,910$ \\
\hline 2007 & 775.511 & 55.441 & 13,988 & 476.714 & 96.114 & 4,960 & & & \\
\hline 2008 & 966.658 & 60.476 & 15,984 & 428.085 & 104.467 & 4,098 & -197.612 & 79.249 & $-2,494$ \\
\hline 2009 & 828.971 & 39.706 & 20,878 & 453.447 & 91.196 & 4,972 & & & \\
\hline 2010 & 876.553 & 66.004 & 13,280 & 467.921 & 151.610 & 3,086 & & & \\
\hline
\end{tabular}

Modelo con Variable Dicotómica: Grupo controlado (HbA1c < 7\%) y no controlado (HbA1 > 10,9\%)

\begin{tabular}{|c|c|c|c|c|c|c|c|c|c|}
\hline \multirow[t]{2}{*}{ Año } & \multicolumn{3}{|c|}{ Constante } & \multicolumn{3}{|c|}{ HbA1c } & \multicolumn{3}{|c|}{ Sexo } \\
\hline & B & Std. Error & $\mathbf{t}$ & B & Std. Error & $\mathbf{t}$ & B & Std. Error & $\mathbf{T}$ \\
\hline 2005 & 943.101 & 152.064 & 6,202 & 689.067 & 279.106 & 2,469 & -439.844 & 201.286 & $-2,185$ \\
\hline 2006 & 1.047 .609 & 54.798 & 19,117 & 284.902 & 122.970 & 2,317 & -290.412 & 72.692 & $-3,995$ \\
\hline 2007 & 775.511 & 56.854 & 13,640 & 501.819 & 154.622 & 3,245 & & & \\
\hline 2008 & 955.420 & 63.587 & 15,663 & 528.432 & 193.787 & 2,727 & -176.404 & 86.243 & $-2,045$ \\
\hline 2009 & 828.970 & 39.857 & 20,799 & 627.305 & 194.238 & 3,230 & & & \\
\hline 2010 & 876.553 & 69.873 & 12,545 & 787.242 & 303.538 & 2,594 & & & \\
\hline
\end{tabular}

Tabla 3. Estimación de gastos promedios en salud (2005-2010) para pacientes diabéticos compensados y no compensados del Hospital del Cobre. Según modelos de correlación (valores en $\$$ de junio de 2011)

\begin{tabular}{|ccccccccc|}
\hline Modelo & \multicolumn{2}{c}{ Compensado } & \multicolumn{2}{c}{ No compensado } & \multicolumn{2}{c|}{ Mayor } & \multicolumn{2}{c|}{ Mayor gasto (\%) } \\
& Hombre & Mujer & Hombre & Mujer & gasto & Hombre & Mujer \\
\hline 1 & 749.873 & 934.281 & 1.150 .791 & 1.335 .200 & 400.919 & $53,5 \%$ & $42,9 \%$ \\
2 & 765.304 & 917.133 & 1.222 .827 & 1.374 .656 & 457.523 & $59,8 \%$ & $49,9 \%$ \\
\hline 3 & 753.417 & 904.527 & 1.323 .212 & 1.474 .322 & 569.795 & $75,6 \%$ & $63,0 \%$ \\
\hline
\end{tabular}

Modelo 1 con Variable Dicotómica: Grupo controlado ( $\mathrm{HbA} 1 \mathrm{c}<7 \%$ ) y no controlado (HbA1 > 6,9\%). Modelo 2 con Variable Dicotómica: Grupo controlado ( $\mathrm{HbA1c}<7 \%$ ) y no controlado (HbA1 > 8,9\%). Modelo 3 con Variable Dicotómica: Grupo controlado (HbA1c < 7\%) y no controlado (HbA1 > 10,9\%). 
ellas (macro y micro vasculares, o hipertensión), se han reportado incrementos de $250 \%$ en el costo medio por paciente, principalmente a expensas de costos hospitalarios, en donde este incremento llega a $550 \%{ }^{23,32,33}$. En nuestro estudio la mayor diferencia reportada en gasto fue entre los grupos con mayor diferencia en el control metabólico, variando entre $20 \%$ y $100 \%$, según año. El control metabólico y la presencia de complicaciones son variables distintas, y es razonable que estas diferenciales de gastos sean disímiles, sin embargo, mantienen tendencias similares.

La variable edad no resultó relevante en la diferencia del gasto en salud entre pacientes compensados y no compensados. Debido a que los gastos en salud en adultos mayores es mayor, y en aumento en las últimas décadas, puede inferirse que la compensación entre los mayores pudiese aportar más, en términos absolutos, al ahorro de recursos en salud.

El gasto en pacientes masculinos diabéticos no controlados es $54 \%$ superior al gasto de pacientes controlados, mientras que en mujeres este incremento es $43 \%$. Cuando se compara el grupo controlado con pacientes diabéticos no controlados con niveles de descompensación "moderados" (HbAlc > 8,9\%) los incrementos en el gasto se elevan a $60 \%$ en hombres y a $50 \%$ en mujeres; cuando se comparan con descompensaciones "graves" (HbAlc $>10,9 \%$ ) el gasto es mayor en $76 \%$ y $63 \%$, respectivamente. Estas diferencias por sexo en las cifras estimadas, se explican fundamentalmente porque la base de gasto para nuestro modelo, es menor en hombres que en mujeres, lo cual es concordante con la realidad nacional: la tasa de uso de servicios sanitarios en mujeres es 1,5 veces mayor ${ }^{49}$.

Algunos hallazgos específicos durante 2005 y 2006 pudieran estar influidos por el proceso de normalización y validación del llenado de datos, sin embargo, al modelar no hubo variaciones observables, pudiendo sólo disminuir el efecto de alguna variable en el modelo general.

En síntesis, nuestros resultados son consistentes con la experiencia internacional que muestra que los pacientes diabéticos no controlados generan una mayor presión sobre el gasto en salud.

Este estudio presenta al menos 3 limitaciones:

Primera, la población evaluada es altamente seleccionada y el sistema sanitario que utilizan presenta características particulares. Se trata en general de personas laboralmente activas (o recientemente retirados del ciclo laboral) y sus cargas, que presenta ingresos económicos superiores al promedio del país. Es reconocido que el nivel socioeconómico determina diferencias tanto en prevalencia como en control metabólico, hecho apoyado por encuestas nacionales ${ }^{4,5}$. Además, probablemente presentan mejor acceso a prestaciones sanitarias. El HDC provee un seguimiento activo y sistemático de enfermería, determinando diagnósticos tempranos y manejo adecuado de complicaciones; y dispone de un arsenal farmacológico vasto. Es difícil extrapolar directamente estos resultados a otros subsistemas de salud, $u$ otras poblaciones.

Segunda, la información utilizada no comprende la totalidad del gasto en salud de los pacientes. Cirugías mayores relacionadas con DM, eventos de baja frecuencia pero de alto costo, han sido omitidas en este estudio, al igual que derivaciones fuera del hospital. Se suma a ello el período relativamente corto del estudio, que no permite relacionar, incluso poblacionalmente, el buen control con el retraso de la aparición de estas complicaciones, como cirugías cardiacas o grandes amputaciones.

Finalmente, el estudio sólo incluye lo que se considera en la literatura como costos directos en salud. Los costos indirectos, tales como productividad laboral, licencias médicas o ausentismo, no forman parte del ámbito del estudio.

Con los resultados obtenidos, se pueden realizar algunos cálculos simples e ilustrativos, de cómo impactaría un mejor control de los pacientes diabéticos en el gasto en el sistema de salud. En ningún caso estos guarismos indican causalidad, ya que el diseño del estudio no es adecuado para establecerla; no obstante, si revelan asociación.

Un ejemplo es estimar el ahorro de recursos que se habría generado en el período con un mejor control metabólico. Si se toma como base el gasto promedio de 2010, y la estructura de pacientes diabéticos de 2005, se estima que el ahorro generado por un mejor control, año a año, de pacientes durante el período 2005-2010 sería aproximadamente $\$ 355$ millones (US\$757.000).

Otro ejercicio es suponer cuánto disminuiría el gasto en salud si todos los pacientes estuvieran compensados. Si consideramos a todos los pacientes de cada año al valor promedio del gasto de cada año, y lo comparamos con la valorización al menor 
gasto en salud medio del tramo de HbAlc menores a 7,0\%, se obtiene una reducción anual promedio de $\$ 308$ millones (US\$657.000). En el período bajo estudio el ahorro correspondería aproximadamente $\$ 1.850$ millones (US\$3,3 millones).

Estos resultados debiesen llamar la atención de profesionales de salud y autoridades sectoriales sobre la necesidad de reforzar estrategias tendientes a mejorar el control metabólico de pacientes diabéticos. El alto impacto se produciría no sólo sobre la calidad de vida de las personas, sino sobre el gasto del sistema de salud.

\section{Referencias}

1. Wild S, Roglic G, Green A, Sicree R, King H. Global prevalence of diabetes: estimates for the year 2000 and projections for 2030. Diabetes Care 2004; 27(5): 104753.

2. Whiting DR, Guariguata L, Weil C, Shaw J. IDF diabetes atlas: global estimates of the prevalence of diabetes for 2011 and 2030. Diabetes Res Clin Pract 2011; 94 (3): 311-21.

3. World Health Organization. Media Centre, Fact Sheet. Diabetes. Disponible en: http://www.who.int/mediacentre/factsheets/fs312/en/index.html. [Consultado el 15 de abril de 2013].

4. Ministerio de Salud. Informe Encuesta Nacional de Salud 2009-2010. Santiago de Chile; 2011.

5. Ministerio de Salud. Informe Encuesta Nacional de Salud 2003. Santiago de Chile; 2004.

6. Superintendencia de Salud. Casos GES (AUGE) Acumulados a diciembre 2011. Informe Trimestral, 2012. Disponible en: http://www.supersalud.gob.cl/documentacion/569/w3-article-7543.html [Consultado el 15 de abril de 2013].

7. Ministerio de Salud. Departamento de Estadísticas e Información de Salud. Población en control por diabetes mellitus según sexo y grupos de edad, por región y servicio de salud, SNSS diciembre 2010. Disponible en (Acceso restringido): http://intradeis.minsal.cl/Reportesrem/2010/PBC_PSCV_DM_2/PBC_PSCV_DM_2. aspx. [Consultado el 15 de abril de 2013].

8. American Diabetes Association. Standards of medical care in diabetes-2006. Diabetes Care 2006; 29 Suppl 1: S4-42.

9. Spellman CW. Insulin therapy for maximal glycemic control in type 2 diabetes mellitus. J Am Osteopath Assoc 2007; 107 (7): 260-9.

10. Ministerio de Salud. Guía Clínica Diabetes Mellitus tipo
2. Serie Guías Clínicas Minsal 1a edición. Santiago de Chile, 2006. No XX.

11. American Diabetes Association. Introduction: The American Diabetes Association's (ADA) evidence-based practice guidelines, standards, and related recommendations and documents for diabetes care. Diabetes Care 2012; 35 Suppl 1: S1-2.

12. World Health Organization. Use of Glycated Haemoglobin (HbAlc) in the Diagnosis of Diabetes Mellitus, in Abbreviated Report of a WHO Consultation, WHO, Editor 2011.

13. Ministerio de Salud. Departamento de Estadísticas e Información de Salud. Población en control por diabetes mellitus con HbA1c menor a 7\%, según sexo y grupo de edad, por región y servicio de salud, SNSS diciembre 2009. Disponible en (acceso restringido): http://intradeis.minsal.cl/Reportesrem/2010/PBC_PSCV_META_ cas_2/PBC_PSCV_META_cas_2.aspx. [Consultado el 15 de abril de 2013].

14. Universidad Mayor . Escuela de Salud Pública. Evaluación Sanitaria de las Intervenciones GES. I. Diabetes Mellitus tipo 2. Informe Final, 2010, Ministerio de Salud: Santiago de Chile.

15. American Diabetes Association. Economic consequences of diabetes mellitus in the U.S. in 1997. Diabetes Care 1998; 21(2): 296-309.

16. Grant RW, Pirraglia PA, Meigs JB, Singer DE. Trends in complexity of diabetes care in the United States from 1991 to 2000. Arch Intern Med 2004; 164 (10): 1134-9.

17. Bolin K, Gip C, Mork AC, Lindgren B. Diabetes, healthcare cost and loss of productivity in Sweden 1987 and 2005 -a register-based approach. Diabet Med 2009; 26 (9): 928-34.

18. Narayan KM, Gregg EW, Fagot-Campagna A, Engelgau MM, Vinicor F. Diabetes-a common, growing, serious, costly, and potentially preventable public health problem. Diabetes Res Clin Pract 2000; 50 Suppl 2: S77-84.

19. Przywara B, Costello D. Health-Care Expenditure Projections: Results, Policy Conclusions and Recommendations for Future Work. Economic Policy Committee and the European Commission. Sin año. Disponible en: http://www.bancaditalia.it/studiricerche/convegni/atti/ fiscal_sustainability/session_3/Przywara_Costello.pdf. [Consultado el 15 de abril de 2013].

20. Schoenmaeckers R, Kotowska I. Population ageing and its challenges to social policy. Study prepared for the European Population Conference 2005. In Population Studies 2005, Council of Europe Publishing.

21. Przywara, B. Projecting future health care expenditure at European level: drivers, methodology and main results. Economic Papers 417. July 2010. 
22. Selby JV, Ray GT, Zhang D, Colby CJ. Excess costs of medical care for patients with diabetes in a managed care population. Diabetes Care 1997; 20 (9): 1396-402.

23. French MT, Mundt MP, Fleming M, Zavala SK. The cost of medical care for patients with diabetes, hypertension and both conditions: does alcohol use play a role? J Intern Med 2005; 258 (1): 45-54.

24. Kapur, A. Economic analysis of diabetes care. Indian J Med Res 2007; 125 (3): 473-82.

25. Fraze T, Jiang HJ, Burgess J. Hospital Stays for Patients with Diabetes, 2008: Statistical Brief \#93. 2010.

26. Russo CA, Jiang HJ. Hospital Stays among Patients with Diabetes, 2004: Statistical Brief \#17. 2006.

27. Francis $\mathrm{BH}$, Song $\mathrm{X}$, Andrews LM, Purkayastha D, Princic N, Sedgley R et al. Progression to type 2 diabetes, healthcare utilization, and cost among pre-diabetic patients with or without comorbid hypertension. Curr Med Res Opin 2011; 27 (4): 809-19.

28. Icks A, Rathmann W, Haastert B, Gandjour A, Holle R, John J, et al. Clinical and cost-effectiveness of primary prevention of Type 2 diabetes in a 'real world' routine healthcare setting: model based on the KORA Survey 2000. Diabet Med 2007; 24 (5): 473-80.

29. O’Brien JA, Shomphe LA, Kavanagh PL, Raggio G, Caro JJ. Direct medical costs of complications resulting from type 2 diabetes in the U.S. Diabetes Care 1998; 21 (7): 1122-8.

30. Ray GT, Collin F, Lieu T, Fireman B, Colby CJ, Quesenberry CP, et al. The cost of health conditions in a health maintenance organization. Med Care Res Rev 2000; 57 (1): 92-109.

31. Brandle M, Zhou H, Smith BR, Marriott D, Burke R, Tabaei BP et al. The direct medical cost of type 2 diabetes. Diabetes Care 2003; 26 (8): 2300-4.

32. Williams R, Van Gaal L, Lucioni C, Board C-A. Assessing the impact of complications on the costs of Type II diabetes. Diabetologia 2002; 45 (7): S13-7.

33. Sachidanandaa A, Geok Linc J, Ushab A. Health care cost incurred by patients of diabetes mellitus in a tertiary care hospital setting in Coastal Karnataka discrict. J Global Pharma Technol 2010; 2 (6): 8-12.

34. Ramsey SD, Newton K, Blough D, McCulloch DK, Sandhu N, Wagner EH. Patient-level estimates of the cost of complications in diabetes in a managed-care population. Pharmacoeconomics 1999; 16 (3): 285-95.

35. Oliva J, Lobo F, Molina B, Monereo S. Direct health care costs of diabetic patients in Spain. Diabetes Care 2004; 27 (11): 2616-21.

36. Zhang P, Zhang X, Betz Brown J, Vistisen D, Sicree R, Shaw J, et al. Economic impact of Diabetes, IDF Diabetes Atlas, 2009.
37. The Diabetes Control and Complications Trial Research Group. The effect of intensive treatment of diabetes on the development and progression of long-term complications in insulin-dependent diabetes mellitus. $\mathrm{N}$ Engl J Med 1993; 329 (14): 977-86.

38. UK Prospective Diabetes Study (UKPDS) Group. Intensive blood-glucose control with sulphonylureas or insulin compared with conventional treatment and risk of complications in patients with type 2 diabetes (UKPDS 33). Lancet 1998; 352 (9131): 837-53.

39. UK Prospective Diabetes Study (UKPDS) Group. Effect of intensive blood-glucose control with metformin on complications in overweight patients with type 2 diabetes (UKPDS 34). Lancet 1998; 352(9131): 854-65.

40. UK Prospective Diabetes Study Group. Tight blood pressure control and risk of macrovascular and microvascular complications in type 2 diabetes: UKPDS 38. BMJ 1998; 317 (7160): 703-13.

41. Early Treatment Diabetic Retinopathy Study Research Group. Early photocoagulation for diabetic retinopathy. ETDRS report number 9. Ophthalmology 1991; 98(5 Suppl): 766-85.

42. Goldberg RB, Mellies MJ, Sacks FM, Moye LA, Howard BV, Howard WJ, et al. Cardiovascular events and their reduction with pravastatin in diabetic and glucoseintolerant myocardial infarction survivors with average cholesterol levels: subgroup analyses in the cholesterol and recurrent events (CARE) trial. The Care Investigators. Circulation 1998; 98 (23): 2513-9.

43. Sidorov J, Gabbay R, Harris R, Shull RD, Girolami S, Tomcavage J, et al. Disease management for diabetes mellitus: impact on hemoglobin Alc. Am J Manag Care 2000; 6 (11): 1217-26.

44. Rothman RL, Malone R, Bryant B, Shintani AK, Crigler B, Dewalt DA, et al. A randomized trial of a primary care-based disease management program to improve cardiovascular risk factors and glycated hemoglobin levels in patients with diabetes. Am J Med 2005; 118 (3): 276-84

45. Norris SL, Nichols PJ, Caspersen CJ, Glasgow RE, Engelgau MM, Jack L, et al. The effectiveness of disease and case management for people with diabetes. A systematic review. Am J Prev Med 2002; 22 (4 Suppl): 15-38.

46. Sidorov J, Shull R, Tomcavage J, Girolami S, Lawton N, Harris R. Does diabetes disease management save money and improve outcomes? A report of simultaneous short-term savings and quality improvement associated with a health maintenance organization-sponsored disease management program among patients fulfilling health employer data and information set criteria. Diabetes Care 2002; 25 (4): 684-9. 
Costos en salud en pacientes diabéticos con mal control - R. Lenz et al

47. Berg GD, Wadhwa S. Diabetes disease management in a community-based setting. Manag Care 2002; 11 (6): 42, 45-50.

48. Beaulieu N, Cutler D, Ho K, Isham G, Lindquist T, Nelson A, et al. The Business Case for Diabetes Disease Management for Managed Care Organizations. Forum for Health Economics \& Policy, 2006. Frontiers in Health Policy Research. The Berkeley
Electronic Press, 2006. Issue 9, Vol 1, Article 1. Disponible en: http://www.health.state.mn.us/ divs/hpsc/hep/transform/081607 documents/ businesscasediabetesdiseasemanagement.pdf. [Consultado el 15 de abril de 2013].

49. Vega J, Bedregal P, Jadue L, Delgado I. [Gender inequity in the access to health care in Chile]. Rev Med Chile 2003; 131 (6): 669-78. 